\title{
Do asthmatics suffer bronchoconstriction during rapid eye movement sleep?
}

\author{
C M SHAPIRO， J R CATTERALL， I MONTGOMERY， G M RAAB， N J DOUGLAS
}

\begin{abstract}
Many patients with asthma are troubled by nocturnal wheeze. The cause of this symptom is unknown, but sleep is an important factor. A study was carried out to determine whether nocturnal bronchoconstriction is related to any specific stage of sleep. Eight asthmatics with nocturnal wheeze and eight control subjects performed forced expiratory manoeuvres immediately after being woken from rapid eye movement (REM) or non-REM sleep, wakings being timed to differentiate temporal effects from those related to the stage of sleep. The control subjects showed no significant temporal bronchoconstriction or bronchoconstriction related to the stage of sleep. All patients showed bronchoconstriction overnight, the mean peak expiratory flow rate falling from 410 (SEM 50) $\mathrm{l} / \mathrm{min}$ before sleep to 186 (49) $\mathrm{l} / \mathrm{min}$ after sleep. After the patients had been woken from REM sleep the forced expiratory volume in one second was on average $300 \mathrm{ml}$ lower $(p<0.02)$ and peak expiratory flow rate $45 \mathrm{l} / \mathrm{min}$ lower $(p<0.03)$ than after they had been woken from non-REM sleep. As wakenings from REM sleep were 21 (8) minutes later in the night than those from non-REM sleep multivariate analysis was performed to differentiate temporal effects from those related to the stage of sleep. This showed that the overnight decreases in forced expiratory volume in one second and peak expiratory flow rate were significantly related both to time and to REM sleep.

This study suggests that asthmatics may suffer bronchoconstriction during REM sleep.
\end{abstract}

Royal Edinburgh Hospital, Edinburgh EH10 5HF

C M SHAPIRO, PHD, MRCPSYCH, senior registrar, department of psychiatry J R CATTERALL, MRCP, senior registrar, department of respiratory medicine I MONTGOMERY, MA, lecturer, department of psychiatry

G M RAAB, MSC, lecturer, department of medical statistics

N J DOUGLAS, MD, FRCPED, senior lecturer, department of respiratory medicine

Correspondence to: Dr Shapiro.

\section{Introduction}

Nocturnal wheeze is common in patients with asthma, and many exhibit falls in forced expiratory flow rates overnight. ${ }^{2}$ Furthermore, deaths and respiratory arrests from asthma, though rare, tend to occur at night. ${ }^{3.5}$ The mechanism of nocturnal bronchoconstriction is obscure ${ }^{5 t}$ : although circadian falls in circulating corticosteroids and catecholamine concentrations may perhaps be causative, ${ }^{7.9}$ these seem unlikely to be the only explanation as treatment to raise nocturnal concentrations to or above daytime concentrations does not abolish nocturnal bronchoconstriction..$^{1 / 1011}$

An alternative possibility is that bronchoconstriction may result directly from sleep. Shift workers with nocturnal asthma find that when they change shifts their bronchoconstriction occurs when they are asleep rather than at night,,$^{1213}$ and sleep may be a prerequisite for overnight bronchoconstriction in some asthmatics. $121+15$ Sleep influences the respiratory system and can lead to obstruction of the upper airway ${ }^{16}$ and increased hypoxia in patients with hypoxic lung disease. ${ }^{17-19}$ Studies of animals have suggested that the muscle tone of the airway varies during rapid eye movement (REM) sleep ${ }^{20}$; we therefore wondered whether in asthmatics, who have a notably labile bronchomotor tone, the calibre of the airway might be altered as a result of REM sleep. This study was designed to determine whether there was any difference in airway calibre between REM and non-REM sleep in adult asthmatics and to compare any such difference with underlying circadian changes.

\section{Subjects and methods}

We studied eight patients with asthma (five men, three women; mean age 30 (range 15-54) years); all were within $15 \%$ of their ideal body weight. ${ }^{21}$ All had reversible airways obstruction with at least one normal value for forced expiratory volume in one second within the past year. The lowest forced expiratory volume in one second recorded in the year before the study ranged from 0.8 to 2.9 litres and the highest from 2.8 to 3.9 litres. Seven patients yielded positive results on skin prick tests to Demodex pteromyssimus or grass pollens, or both. All complained of nocturnal wheeze and had documented "morning dips" in peak expiratory flow rates. None had had an acute attack of asthma for six weeks before the study. Two inhaled sodium cromoglycate regularly and two took oral prednisolone ( 5 and $10 \mathrm{mg}$ daily). All inhaled $\beta_{2}$ sympathetic agonists, but these were withheld for six hours 
before the study. Each patient was matched for age and sex with a healthy subject with no history of respiratory disease, who was studied on the same night.

Each subject slept for three non-consecutive nights in the sleep laboratory. The first night was to acclimatise the subjects, and data from only the second and third nights are reported. On each night the subjects were woken on three occasions and immediately performed forced expiratory manoeuvres. The timing of their wakenings was designed to differentiate temporal changes in airway calibre from those related to stages of sleep. On night $A$ the patients were woken 10 minutes after the end of the first period of REM sleep and again 60 minutes later, before the start of the next REM period (fig 1). On night $B$ the patients were woken 60 minutes after the onset of sleep (before the first period of REM sleep) and five to seven minutes after the start
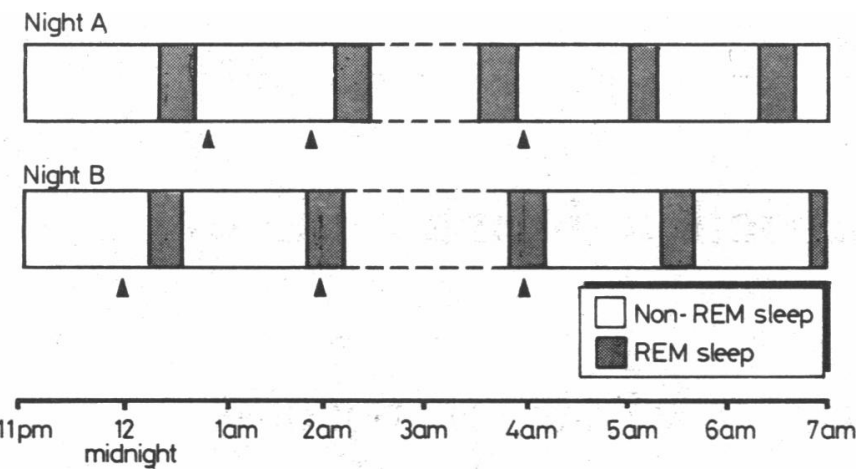

FIG 1-Schematic diagram of times of wakening (arrows) on second and third nights. Broken lines indicate second randomisation, in which wakenings from REM and non-REM sleep were random.
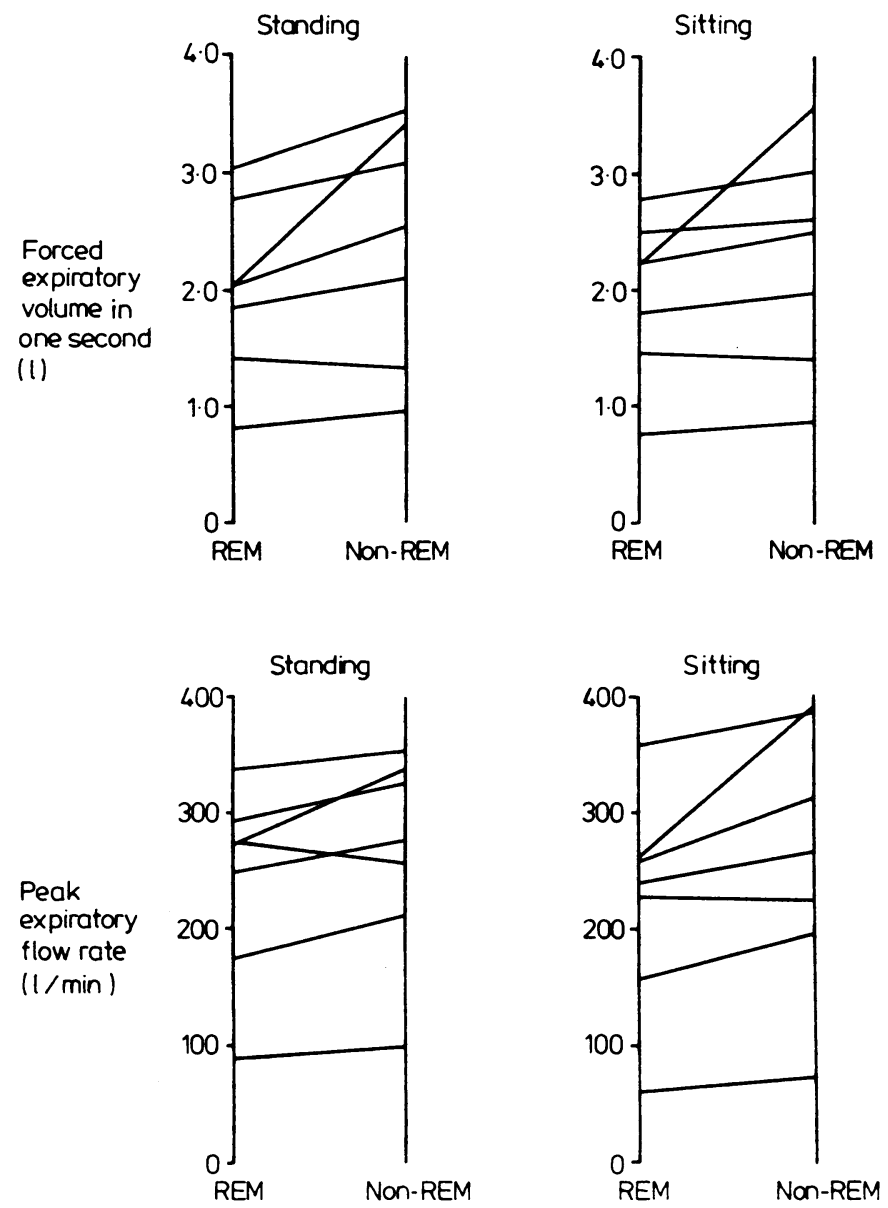

FIG 2-Peak expiratory flow rate and forced expiratory volume in one second in postures indicated after patients were woken from REM and non-REM sleep. Each line represents average of three time matched comparisons (five patients) or two time matched awakenings (two). For all four measures $\mathrm{p}<0.05$. of the second period. The order of night $A$ and night $B$ was randomised so that half the patients did night $A$ first and half night $B$. The third wakening was from the first period of REM sleep after 4 am on one night and the first $\underline{\underline{W}}$ period of non-REM sleep after 4 am on the other night; the order of these $<$ nights was randomised independently of the night $\mathrm{A} /$ night $\mathrm{B}$ randomisation. $\mathrm{\Phi}$

On both nights forced expiratory volume in one second, forced vital capacity, and peak expiratory flow rate were also measured at $930 \mathrm{pm}$; $11 \mathrm{pm}$, just before lights out; immediately on the final awakening in the morning; and one hour thereafter. On each of these occasions and after the imposed wakenings the peak expiratory flow rate was measured first with the subject supine, then forced expiratory volume in one second was measured ${ }^{\text {of }}$ with the subject sitting on the bed, and finally both peak expiratory flow rate $\mathrm{I}_{2}$ and forced expiratory volume in one second were measured while the subject was standing. All measurements were made in duplicate, except for the $\overrightarrow{\vec{F}}$ standing forced expiratory volume in one second, which was measured in triplicate, and on each occasion the highest value was taken.

Each subject slept in a quiet, darkened single room on each night of the study. The electroencephalogram was recorded by two midline fronto- $\frac{T}{T}$ parietal electrodes, the electro-oculogram by four electrodes above and $\stackrel{\mathbb{\Phi}}{\Omega}$ outside the outer canthi, and the electromyogram by two submental electrodes. The stage of sleep was analysed by standard criteria. ${ }^{22}$

The subjects gave their informed consent, and the study was approved by $\overrightarrow{0}$ the hospital's ethical committee.

Results are expressed as means (SEM). Statistical analysis was by $\vec{\omega}$ Student's $t$ test for paired comparisons. The Wilcoxon signed rank test was used for the pooled information depicted in figure 2. A log transformation 3 was used for the time that the subjects took to fall back to sleep. All data obtained when the patients were woken were analysed by multiple $\mathbb{N}$ regression methods to enable the effects of the stage of sleep and time to be o separated..$^{23}$

\section{Results}

\section{NORMAL SUBJECTS}

In the eight normal subjects there was no bronchoconstriction overnight (for example, the peak expiratory flow rate supine was 413 (SEM 38) $1 / \mathrm{min}$ ₹ before sleep and $400(40) \mathrm{l} / \mathrm{min}$ after sleep in the controls; in the asthmatic $\vec{\varphi}$ patients the peak expiratory flow rate standing was 410 (50) $1 / \mathrm{min}$ before ${ }_{0}^{\circ}$ sleep and 186 (49) $\mathrm{l} / \mathrm{min}$ after sleep).

There was no difference in time matched REM and non-REM measures in the normal subjects (for example, forced expiratory volume in one second standing was $3.5(0.36)$ litres and $3.5(0.39)$ litres on waking from REM and $\bar{O}$ non-REM sleep respectively). The controls spent significantly more time in stage 2 sleep (mean 234 minutes, range 190-282) than the patients (mean 201 Ф (range 167-234) minutes; $p<0 \cdot 05$ ). The controls showed more frequent shifts into stage 3 sleep (on average 18.9 occasions a night) compared with the patients $(14.4$ occasions a night; $p<0.05)$.

\section{ASTHMATICS' SLEEP}

The patients were more wakeful (81 (9) $v 64(6) \mathrm{min}$ ) on the acclimatisation night with less REM sleep $\left(67\right.$ (7) $v 87$ (11) min) than on the subsequent $\frac{5}{3}$ nights $(\mathrm{p}<0.01)$, but there was no difference in duration (396 (16) v $400 \%$ (13) min) or quality of sleep between the second and third nights. The $O$ average times taken to fall back to sleep after wakenings from stage 2 and REM sleep were similar (6 (2) $\min ; n=80$ ), but the times taken after $ᄋ$ wakenings from stage 3 and stage 4 sleep were shorter $(3$ (1) $\min ; n=8$; N $p<0.05$ ). One patient, however, stayed awake after the second wakening on $\rightarrow$ night $A$ and fell asleep only after using his bronchodilator. His subsequent $\frac{D}{2}$ data from that night were excluded from the analysis.

\section{FORCED EXPIRATORY MANOEUVRES} Every patient experienced bronchoconstriction overnight. All measure- $\bullet$ and standing, forced expiratory volume in one second both sitting and $\stackrel{\mathscr{O}}{+}$ standing) decreased significantly more (range of $p$ values $0.04-0.01$ ) when $T$ there had been intervening periods of REM sleep (night $B$ ) than when there $O$

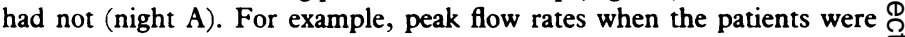
standing fell by 39 (9) $\mathrm{l} / \mathrm{min}$ when no REM sleep had intervened but by $120 \stackrel{\mathbb{D}}{ }$ (33) $\mathrm{l} / \mathrm{min}(\mathbf{p}<0.02)$ when REM sleep had occurred. The intervals containing REM sleep, however, were significantly longer $(190(26) \mathrm{min})$ than the intervals without any REM sleep (61 (3) $\mathrm{min})$. Thus this comparison 8 cannot fully distinguish effects of stages of sleep from those of time.

To examine predicted effects of stages of sleep rather than temporal effects we compared the measurements of forced expiratory volume in one second 
and peak expiratory flow rate made after patients were woken from REM sleep with those made within 70 minutes of the same time on a different night when the patients were woken from non-REM sleep (time matched measurements). When the two or three time matched measures for each subject were averaged, all forced expiration after wakenings from REM sleep was significantly lower $(p<0.05)$ than that after wakenings from nonREM sleep (fig 2). The data obtained from this time matched comparison of wakenings from REM and non-REM sleep were plotted against time of night (fig 3). The overall decrease in forced expiratory volume in one second overnight was much greater than the difference in forced expiratory volume in one second between REM and non-REM sleep.

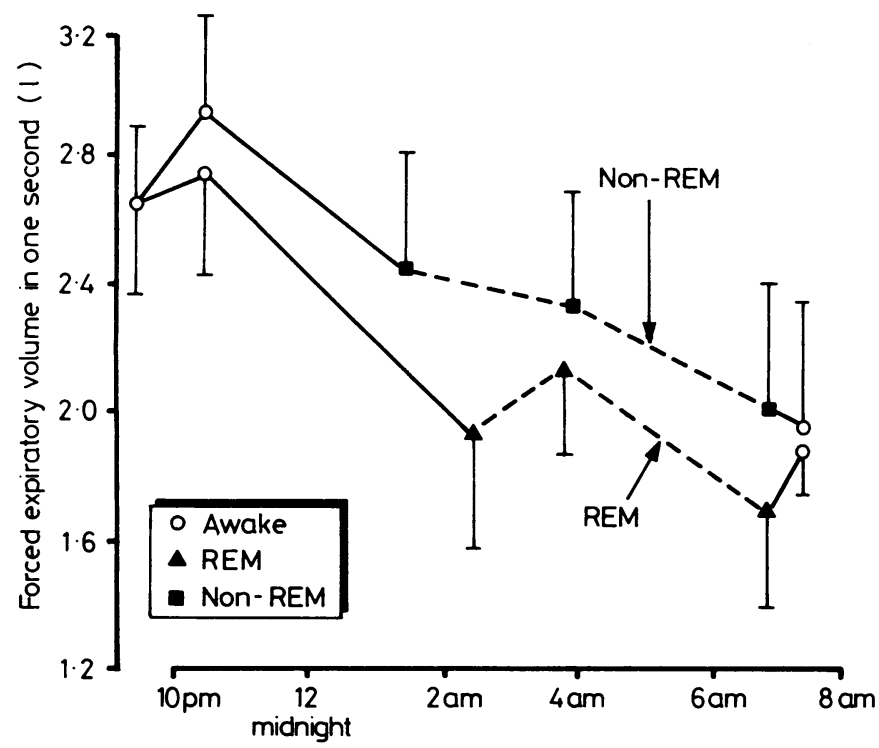

FIG 3-Forced expiratory volume in one second measured standing after wakening from REM or non-REM sleep at times indicated; values are means (SEM) in seven patients using data from both night $A$ and night $B$. Solid lines join data obtained in the subjects on the same night; broken lines indicate when data were from different nights owing to the two separate randomisation procedures.

These time matched wakenings from non-REM sleep occurred an average of 21 (8) minutes (range -4 to 69 minutes) earlier than those from REM sleep and thus cannot fully differentiate sleep effects from temporal effects. The above analyses of time matched values do not use all the data collected to separate the effects on forced expiration of time of night from those due to the stage of sleep. This was achieved by fitting a regression model to all the data obtained in each patient. The model was built up by including the most important influences on the measurements of forced expiratory volume in one second and peak expiratory flow rate in each posture. In the final model (table) for all four measurements of forced expiration the most important influence was that of time $(p<0.01)$. Taken over the whole night the effect of REM sleep was significant for one of the respiratory measures (peak expiratory flow rate lying, $p<0.05)$ but not significant $(0.1>p>0.05)$ for the others. When a model was fitted that allowed the influence of REM sleep to

Effect of rapid eye movement sleep on bronchoconstrition: fitted values of the variables in the linear equation derived from all data from the eight as asthmatics, $Y=P_{i}-t S_{i}-D_{R E M}+(t-2) S_{R E M}{ }^{\star}$

\begin{tabular}{|c|c|c|c|c|}
\hline $\mathrm{Y}$ & $\begin{array}{c}\mathbf{P}_{\mathrm{i}} \\
(\text { mean }(\text { range }))\end{array}$ & $\begin{array}{c}\mathrm{S}_{\mathrm{i}} \\
\text { (mean (range) })\end{array}$ & $\mathrm{D}_{\text {REM }}$ & $S_{\text {REM }}$ \\
\hline \multicolumn{5}{|l|}{ PEFR: } \\
\hline Standing & $362(200-534) \mathrm{l} / \mathrm{min}$ & $14(-2$ to 50$) \mathrm{l} / \mathrm{min} / \mathrm{h}$ & $821 / \min$ & $19 \mathrm{l} / \mathrm{min} / \mathrm{h}$ \\
\hline Sitting & $322(162-454) \mathrm{l} / \mathrm{min}$ & $13(2-38) 1 / \mathrm{min} / \mathrm{h}$ & $741 / \min$ & $211 / \mathrm{min} / \mathrm{h}$ \\
\hline \multicolumn{5}{|l|}{$\mathrm{FEV}_{1}:$} \\
\hline Standing & $2 \cdot 72(1.61-3.72) 1$ & $0.11(0.02-0.20) \mathrm{l} / \mathrm{h}$ & 0.461 & $0 \cdot 12 \mathrm{l} / \mathrm{h}$ \\
\hline Sitting & $2.58(1.50-3.54) 1$ & $0.10(0.01-0.24) 1 / \mathrm{h}$ & $0 \cdot 391$ & $0 \cdot 10 \mathrm{l} / \mathrm{h}$ \\
\hline
\end{tabular}

$P E F R=$ peak expiratory flow rate

$\mathrm{FEV}_{1}=$ forced expiratory volume in one second

$\star \mathrm{Y}=\mathrm{FEV} \mathrm{V}_{1}$ (or $\mathrm{PEFR}$ ) in each posture; $\mathrm{P}_{\mathrm{i}}=\mathrm{FEV} \mathrm{F}_{1}$ (or $\mathrm{PEFR}$ ) in non-REM sleep at midnight in the ith patient; $t=$ time after midnight; $S_{i}=$ slope of decline in $F E V_{1}$ (or PEFR) with time in the ith patient; $D_{\text {REM }}=$ decrease in $F E V_{1}$ (or PEFR) due to REM sleep at $2 \mathrm{am} ; \mathrm{S}_{\mathrm{REM}}=$ change in effect of REM sleep on FEV, or PEFR with time. decline with time of night, however, this gave a significantly better fit for all four expiratory manoeuvres $(p<0.05)$. The effect of REM sleep was greatest early in the night and declined towards morning for all four measurements.

\section{Discussion}

This study suggests that nocturnal bronchoconstriction in adult asthmatics may be associated with REM sleep but that it is also greatly affected by time. The data confirm that the circadian variation in the calibre of the airway is greater in asthmatics than in normal subjects, ${ }^{\prime}$ and indeed the changes in normal subjects are so small that larger numbers are required to detect it.

It is difficult to distinguish the effects of electroencephalographic stages of sleep on nocturnal events from those of circadian rhythm. Studies of asthmatic shift workers have shown that overnight bronchoconstriction is related to sleep time rather than clock time. ${ }^{12} 13$ Three studies have tried to determine the effects of sleep by keeping asthmatics awake at night, and each has suggested that sleep is important in the pathogenesis of nocturnal asthma. ${ }^{121+15}$ The current investigation extends these observations by establishing that the REM (dreaming) stage of sleep may be associated with bronchoconstriction.

Previous studies have tried to assess whether bronchoconstriction is related to stages of sleep by noting the sleep stage from which asthmatics awoke with attacks. Despite an early suggestion that they woke most often from REM sleep,,$^{24}$ it now seems that such awakenings are not preferentially related to any stage of sleep but are distributed throughout the night in proportion to the time spent in the various stages of sleep. ${ }^{25 \cdot 27}$ These data, however, must be interpreted cautiously as asthmatic attacks were often loosely defined, and it is not known whether the arousal threshold to bronchoconstriction differs between REM and non-REM sleep. Most animal experiments, ${ }^{28}$ and those in man involving nonrespiratory stimuli, ${ }^{29}$ show that it is more difficult to arouse patients from REM than stage 2 sleep, but two recent experiments have shown that arousal in patients with airway occlusion ${ }^{30}$ and with hypercapnia $^{31}$ is more rapid from REM than non-REM sleep. Montplaisir et al noted that the most severe hypoxia and falls in forced expiratory flow rates usually occur in the later hours of sleep, coinciding with the time when most REM sleep occurs, but pointed out that this does not prove any relation between bronchoconstriction and the stage of sleep. ${ }^{27}$

The lower flow rates found after patients were woken from REM sleep might be explained by impaired function of expiratory muscles during REM sleep, which is a stage characterised by voluntary muscle hypotonia. The subjects were, however, fully awake when they performed the expiratory manoeuvres, with normal submental electromyogram tone. Furthermore, the difference in forced expiratory flow rates was seen in each of the four sequential types of forced expiratory flow manoeuvres, and thus there was no evidence that the effect was transient after awakening. Other studies of the performance of voluntary tasks on wakening from different sleep stages suggest that the worst performance occurs on wakening from stages 3 and 4 sleep, with no significant difference between stage 2-from which most of the non-REM awakenings were made-and REM sleep. ${ }^{32}$ Additionally, the normal controls would be expected to show such a performance effect yet did not.

Thus we conclude that the significant effects of REM sleep in asthmatics on forced expiratory manoeuvres reflect alterations in the calibre of the airway. Asthmatics respond more than normal subjects to a wide variety of bronchoconstricting influences, and we suggest that REM sleep is one such stimulus.

REM sleep could cause bronchoconstriction either by a direct onoff effect or by producing an effect that persists after the REM period has finished. The results from the modelling procedure and from the time matched comparison both suggest that REM sleep has a direct effect, but whether it has a persisting effect on airway tone is unclear. We may have underestimated the effect of REM sleep in this study as the decline overnight may partially result from an accumulative effect of periods of REM sleep throughout the night. A decline in airway calibre, however, was also observed between the 
wakenings without intervening REM sleep, suggesting that there is a time effect independent of the effect of REM sleep.

Our suggestion that REM sleep causes bronchoconstriction requires confirmation as we studied relatively few subjects and, by waking them, necessarily interrupted the variable under studynamely, REM sleep. The declining effect of REM sleep later in the night also requires confirmation as this was noted only in retrospect.

There have been no satisfactory measurements of bronchomotor tone in sleeping asthmatics, perhaps because oesophageal intubation is required for conventional measurement, and patients with nocturnal asthma sleep poorly anyway..$^{2 ; 3}$ One study used a tape recording of breath sounds to try to detect bronchoconstriction but found no relation between REM sleep and bronchoconstriction in asthmatic children. ${ }^{26}$ As ventilation is on average lower in REM sleep, ${ }^{24}$ however, wheeze might even decrease in REM sleep despite bronchoconstriction. Nevertheless, our data suggest that REM sleep may be associated with bronchoconstriction.

The mechanism of bronchoconstriction in REM sleep requires elucidation. In dogs the variability in bronchial smooth muscle tone during REM sleep can be abolished by cooling the vagus nerve. ${ }^{29}$

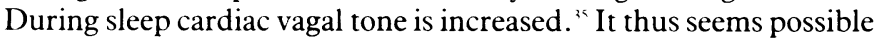
that changes in airway vagal tone related to sleep could lead to bronchoconstriction related to REM sleep and nocturnal asthma, suggesting that inhaled anticholinergic agents warrant further study in this common and important condition.

We thank Professor I Oswald for use of facilities and Mrs C Hoy for technical help. We thank Professor D C Flenley for advice. CMS was supported by Roussel Laboratories Ltd, Paris, during the study.

\section{References}

1 Turner-Warwick $\mathrm{M}$. On observing patterns of airflow obstruction in chronic asthma. Br 7 Dis Chest 1977;71:73-86

2 Connolly CK. Diurnal rhythms and airway obstruction. Br f Dis Chest 1979:73:357-66.

3 Hetzel MR, Clark TGH, Brathwaite MA. Asthma: analysis of sudden deaths and ventilatory arrests in hospital. BrMed 1 1977; i:808-11.

4 Cochrane GM. Clark TGH. A survey of asthma mortality in patients between ages 35 and 64 in the greater London hospitals in 1971. Thorax 1975;30:300-5

5 Douglas NJ. Asthma at night. Clin Chest Med 1985;6:663-74.

7 Soutar CA, Crostello J, Ijaduola O, Turner-Warwick M. Nocturnal and morning asthma. Thorax 1975;30:436-40.
8 Soutar CA, Caruthers M, Pickering CAC. Nocturnal asthma and urinary adrenaline and noradrenaline excretion. Thorax 1977;32:677-83.

Barnes PJ, Fitzgerald G, Brown M, Dollery C. Nocturnal asthma and changes in circulating epinephrine, histamine and cortisol. $N$ Engl f Med 1980;303:263-7.

10 Fairfax AJ, MacNab WR, Davies HJ, Spiro SG. Slow-release oral salbutamol and aminophylline in nocturnal asthma: relation of overnight change in lung function and plasma drug levels. Thorax 1980;35:526-30.

11 Milledge JS, Morris J. A comparison of slow-release salbutamol with slow-release aminophylline in nocturnal asthma. I Int Med Res 1979; 7(suppl 1): 106-10.

12 Hetzel MR, Clark TGH. Does sleep cause nocturnal asthma? Thorax 1979;34:749-54.

13 Connolly CK. The effect of bronchodilators on diurnal rhythms in airway obstruction. Br 7 Dis Chest 1981;75:197-203.

14 Reinhardt D, Schuhmacher P, Fox A, Stemman EA, Wegner F. Comparison of the effect of theophylline, prednisolone and sleep withdrawal on airway obstruction and urinary cyclic AMP/cyclic GMP excretion of asthmatic children with and without nocturnal asthma. Int $\mathcal{f}$ Clin Pharmacol Ther Toxicol 1980;18:399-404.

15 Catterall JR, Rhind GB, Stewart IC, Whyte KF, Shapiro CM, Douglas NJ. Effect of sleep deprivation on overnight bronchoconstriction in nocturnal asthma. Thorax (in press).

16 Guilleminault C, Tilkian A, Dement WC. The sleep apnea syndromes. Annu Rev Med 1976;27:465-84.

17 Koo KW', Sax DS, Snider GL. Arterial blood gases and $\mathrm{pH}$ during sleep in chronic obstructive pulmonary disease. Am F Med 1975:58:663-70

18 Douglas NJ, Calverley PMA, Leggett RJE, Brash HM, Flenley DC, Brezinova V. Transient hypoxaemia during sleep in chronic bronchitis and emphvsema. Lancet 1979;i:1-4.

$19 W_{\text {ynne JW, Block AJ, Hemenway J, Hunt LA, Flick MR. Disordered breathing and oxygen }}$ desaturation during sleep in patients with chronic obstructive lung disease. Am $\mathcal{J}$ Med 1979;66:573-9.

20 Sullivan CE, Zamel N, Kozar LF, Murphy E, Phillipson EA. Regulation of airway smooth muscle tone in sleeping dogs. Am Rev Respir Dis 1979;119:87-99.

21 Documenta Geigy. Scientific tables. 7th ed. Manchester: Geigy Pharmaceuticals Co Ltd, 1970

22 Rechtschaffen A, Kales A, eds. A manual of standardised terminologv, techniques and scorng system for sleep stages of human subjects. Bethesda, Maryland: National Institute of Neurological Disease and Blindness, 1968. NI publication No 204.

23 Draper NR, Smith H. Applied regression analysis. 2nd ed. New York: Wilev, 1980

24 Ravenscroft K, Hartmann EL. The temporal correlation of nocturnal asthmatic attacks in the D state. Psychophysiology 1968;4:396-7

25 Kales A, Beall GN, Bajor GS, Jacobson A, Kales JD. Sleep studies in asthmatic adults: relationship of attacks to sleen stage and time of night. Foumal of Allergv 1968;41:164-73.

26 Kales A, Kales JD, Sly RM, Scharf MB, Tan T, Preston TA. Sleep patterns of asthmatic children: all night electroencephalographic studies. Foumal of Allergy 1970;46:300-8.

27 Montplaisir J, Walsh J, Malo JL. Nocturnal asthma features of attacks, sleep and breathing patterns. Am Rev Respir Dis 1982;125: 18-22.

28 Steriade M. Hobson JA. Neuronal activity during the sleep-waking cycle. Prog Neurobiol 1976;6:155-376

29 Savin JA, Paterson WD, Oswald I. Scratching during sleep. Lancet 1973;ii:296-7.

30 Issa FG, Sullivan CE. Arousal and breathing responses to airway occlusion in healthy sleeping adults. F Appl Physol 1983:55:1113-9.

31 Berthon-Jones $M$, Sullivan $C E$. Ventilation and arousal responses to hypercapnia in normal sleeping adults. F Appl Physiol 1984:57:59-67.

32 Wilkinson RT, Stretton M. Performance after awakening at different times of night. Psycho somatic Science 1971;23:283-5.

33 Catterall JR, Douglas NJ, Calverley PMA, et al. Irregular breathing and hypoxacmia during sleep in chronic stable asthma. Lancet 1982;i:301-44

34 Douglas NJ, White DP, Pickett CK, W'eil JV, Zwillich CW. Respiration during sleep in normal man. Thorax 1982;37:840-4

35 Baust $\mathbf{W}$, Bohnert B. The regulation of heart rate during sleep. Exp Brain Res 1979;7:169-80

Accepted 5 February 1986

\section{YEARS AGO}

The following advertisement appeared, under the heading "Sales by Auction," in the Kensington Express of January 30th:- "Earl's Court House (nearly facing Earl's Court Station). Messrs. John W. Morley and Letts, having been favoured with instructions by Mr. James Whittaker, will sell by auction, on the ground, on Tuesday and Wednesday, February 16th and 17 th, 1886, at 12 o'clock each day, the old building materials of the above historical mansion, the residence of the late John Hunter, the Duke of Richmond, and the Earl of Albemarle, comprising a large quantity of sound floor-boarding, beams, joists, roofing and partitioning, rough boarding, match lining, etc., old staircases, lantern-lights, etc., several tons of lead in hips, ridges, flats, cisterns, and piping, a quantity of wrought and cast iron work in railings, gratings, and balconies, verandahs, guttering, and piping, galvanised iron tanks, zinc work, sheet copper bells, wire, etc., over 120 four and six-panel doors, a large quantity of sashes, casements, frames, shutters, etc., a pair of antique six-panel doors, with beautifully painted panels, numerous old-fashioned fretwork, brass finger-plates and escutcheons, a quantity of tiling, stone coping and paving, the fittings of bath-room, w.c. apparatus, etc., about 100 feet run of glazed corridors, the historical copper, coving, and fittings, used for the purpose of boiling the remains of the Irish Giant, 'Byrne O'Brian,' statuary marble chimney-piece, old and modern grates, ranges, etc., lean-to glass-house, about 50 feet run, a large vinery and vine, about 42 feet run, with flues, staging, etc., a quantity of choice shrubs, fine variegated hollies, and some large hollytrees, suitable for cutting up for turnery-purposes, oak, ash, and elm trees, a quantity of fruit trees and bushes, some splendid lawn-turf, iron hurdles, garden tile edging, etc., and sundries. On view day prior to sale days. Catalogues on the premises, and of the auctioneers, Earl's Court Road, near the racing station." A letter, addressed by Mr. John Merriman, sen., of Kensington, to the editor of the Kensington Express, in reference to a notice of Hunter as an inhabitant of Kensington, was published in that journal on Saturday, January 30th. It shows that the Hunter family mixed with the cream of the intellectual society of the period, including accomplished men of fashion like Horace Walpole. This fact is occasionally overlooked. John Hunter was devoted to science but was no ascetic. Mr. Merriman adds to the notice published in the local journal of the illustrious John Hunter, and his thirty years' residence a Earl's Court, Kensington, a few lines concerning the social receptions held by his wife. Mrs. Anne Hunter was sister of Sir Everard Home, and a very accomplished lady. On Thursdays, at her receptions, might be seen Sir Joseph Banks, Dr. Johnson, David Garrick, Horace Walpole, Sir Joshua Reynolds, Mrs. Montague, Dr. Solander, Mrs. Elizabeth Carter, Oliver Goldsmith, Miss Berry, Benjamin Stillingfleet (who was barrack-master at Kensington), Mme. D'Arblay, Mrs. Chapone, and a host of other artists, authors, and virtuosi. Mrs. Hunter published a volume of poems of considerable merit; she wrote a touching epitaph on her husband, which however, was not allowed by the rector to be placed in St. Martin's-in-theFields Church; and her sweetly simple charming song, 'My mother bids me bind me hair,' set to music by Haydn, is too well known to need comment. Only one of her painted rooms remains in the house shortly to be demolished. The panels to which Mr. Merriman refers are interesting specimens of Mrs. Hunter's work, which is of fair amateur quality. (British

(British Medical Fournal 1886;i:314.) 